\title{
Evaporation in focus
}

To the Editor - The hydrological cycle is expected to accelerate as temperatures rise and the capacity of the air to carry moisture increases. Observational evidence for such acceleration is largely based on trends in precipitation or river discharge, because our capability to observe the third crucial component of the system - the return flow of moisture from the land to the atmosphere via evaporation - is still poor. However, as observational techniques that used to be restricted to research applications are entering operational mode, it is becoming possible to routinely measure evaporation and soil moisture, based on surface and satellite-mounted observation. We can therefore move away from merely closing a water budget, towards considering all the components and dynamics of the hydrological cycle based on observational evidence of all fluxes and states.

The fundamental question of whether evaporation is increasing or decreasing, and, if so, where and when this is occurring, has not yet been answered. Without this information, we cannot claim to understand the global hydrological cycle or its role in global climate. Evaporation is a key process, not only because it directly affects the hydrological cycle, but also because it contributes to the Earth's surface energy balance. Solar radiation absorbed by the Earth's surface fuels evaporation and convective heat flux in different proportions. Changing the ratio of these two processes affects the thermodynamics and, in turn, the dynamics of the atmosphere, and could thereby alter weather and climate.

Traditionally, hydrologists have only inferred evaporation from the difference between precipitation and river discharge from a catchment - two large and variable entities. But for more than ten years now, direct observations of evaporation have routinely been available from the global collaboration Fluxnet ${ }^{1}$, a network of towers that collects measurements on the magnitude and controls of surface evaporation over a wide range of biomes. Efforts are underway to ensure the long-term continuity of these observations in several continents.

Land surface evaporation is limited by the availability of soil moisture. However, surface soil moisture often varies by an order of magnitude over distances as small as a few metres. In situ observation techniques typically sample less than $1 \mathrm{~m}^{2}$, making it hard to collect enough data so that meaningful comparisons can be made, for example, with climate model output on scales of about $100 \mathrm{~km}$. Considerable progress in developing algorithms ${ }^{2}$ to calculate soil moisture from satellite observations of microwave radiation over the past 30 years has the potential to fill this gap. The European Space Agency's recently launched Soil Moisture and Ocean Salinity (SMOS) mission, and NASA's planned Soil Moisture Active and Passive (SMAP) mission can build on these advances and should provide important information on the mechanisms controlling evaporation. In addition, the twin satellites of the Gravity Recovery and Climate Experiment (GRACE) measure the gravitational pull of the Earth below, and can detect changes in the mass of total soil water. This type of observation has revealed the drying and subsequent seasonal rewetting of continents, as well as dwindling reserves of groundwater in areas where it is mined for large-scale irrigation ${ }^{3}$.

At this point in time, our understanding of the coupling of soil moisture and precipitation is still limited and relies heavily on model-based evidence ${ }^{4}$. But the combination of satellite-derived groundwater and soil moisture observations with directly measured evaporation data makes the surface link of the causal chain amenable to detailed investigation. With these measurements in place we will be in a good position to detect and understand the ongoing subtle changes in the global hydrological cycle.

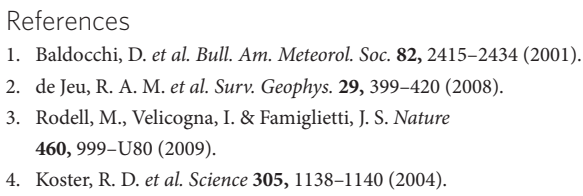

\section{A. J. Dolman and R. A. M. de Jeu}

Department of Earth Sciences, VU University Amsterdam, Boelelaan 1085, $1081 \mathrm{HV}$

Amsterdam, The Netherlands.

e-mail: han.dolman@geo.falw.vu.nl 